\title{
ANÁLISE EXPLORATÓRIA DA COLABORAÇÃO DOS FUNCIONÁRIOS E DO DESEMPENHO DO PLANEJAMENTO COLABORATIVO NA KLABIN S.A.
}

\section{AN EXPLORATORY ANALYSIS FROM EMPLOYEES COLLABORATION FOR PERFORMANCE OF THE COLLABORATIVE PLANNING IN KLABIN S.A.}

ROBERTO GIRO MOORI

Doutor em Engenharia da Produção pela USP.

Professor titular do Programa de Pós-Graduação "Stricto Senso" em Administração da Universidade Presbiteriana Mackenzie.

Área de interesse em pesquisa: Operações, Logística e Gestão de Cadeias de Suprimentos. Rua da Consolação, 896 - São Paulo - SP - CEP $01302-907$ E-mail: rgmoori@mackenzie.com.br

\section{ANTONO CARLOS DOMENEK}

Mestre em Administração de Empresas pela Universidade Presbiteriana Mackenzie.

Gerente de Suprimentos da Klabin S.A.

Área de interesse em pesquisa: Operações, Logística e Gestão de Cadeias de Suprimentos. Rua da Consolação, 896 - São Paulo - SP - CEP $01302-907$ E-mail: ACDomenek@klabin.com.br 


\section{RESUMO}

O planejamento colaborativo tem como foco o compartilhamento de informações estratégicas entre os membros da cadeia produtiva, objetivando desempenho competitivo. Todavia, obter desempenho competitivo depende da colaboração dos funcionários. À vista disso, esta pesquisa de natureza exploratória do tipo estudo de caso investiga o grau de relacionamento entre a colaboração dos funcionários e o desempenho do planejamento colaborativo na empresa brasileira de papel e celulose, a Klabin S. A. O resultado da pesquisa, analisado com dados primários obtidos por meio de entrevistas, mostrou evidência de alto grau de relacionamento entre a colaboração e o desempenho do planejamento colaborativo. Esse resultado foi corroborado na análise dos dados secundários, coletados por meio dos documentos oficiais da empresa. Concluiu-se que, embora houvesse a evidência do relacionamento positivo, esse resultado não pode ser analisado somente com base na colaboração dos funcionários. A análise deve incluir outras variáveis como treinamento e valorização profissional, qualificações técnicas e ferramentas gerenciais, para obter uma melhor acuracidade do grau de relacionamento de cada uma delas no desempenho do planejamento colaborativo.

\section{PALAVRAS-CHAVE}

Comprometimento; Confiança; Trabalho em equipe; Klabin, Gestão da cadeia de suprimentos; Planejamento colaborativo.

\section{ABSTRACT}

The objective of collaborative planning is the strategical information sharing among members of supply chain for competitive advantage. However, to get competitive performance depends on the employees' collaboration. Trying to study more in depth this theme, this article of exploratory nature and study case 
methodology, investigated the level of the relationship between the collaboration of the employees and the collaborative planning performance at the brazilian company of paper and cellulose, Klabin S. A. The research result, analyzed with primary data gotten by interviews, evidenced high relationship level between employees' collaboration and colaborative planning performance. This result was corroborated by secondary data analysis, collected in official documents of the company. Although it had evidences of positive relationship, this results can not be analyzed just by the employees' collaboration. The analysis must include other variables as training and professional valuation, managerial skills programme, techniques and tools for management to obtain a better accuracity of the relationship level in each one of them to collaborative planning performance.

\section{KEYWORDS}

Commitment; Trust; Teamworking; Klabin; Supply chain management; Collaborative planning.

\section{INTRODUÇÃ O}

Com a globalização e as atuais políticas do livre-comércio que permitem que os fatores vitais de produção como o capital, a tecnologia e o conhecimento fluam livremente ao redor do mundo, surgiram vários conceitos administrativos para tratar da integração das cadeias produtivas. No início da década de I990, um dos primeiros conceitos a aparecer sob esse enfoque foi a gestão da cadeia de suprimentos como uma extensão da logística (CHRISTOPHER, I992). Enquanto o gerenciamento da logística buscava a integração interna por meio da otimização dos fluxos dos materiais e da informação, o gerenciamento da cadeia de suprimentos reconhecia que a integração por si só não era suficiente. Era necessário planejar e coordenar o fluxo dos materiais e da informação para fora dos limites da empresa, a fim de incluir os fornecedores e os clientes. Assim, o mercado, a rede de distribuição, o processo de fabricação e as atividades de aquisição teriam que ser integrados de tal modo que a vantagem competitiva fosse alcançada por meio da redução de custos e da melhoria dos níveis de serviços.

O fácil acesso às últimas informações do mercado e a disponibilidade de conhecimentos na internet possibilitaram essa integração. No entanto, isso não ocorreu (WHITE, I999). A necessidade de disponibilizar informações consideradas estratégicas pelas empresas, como níveis de estoques e demanda de curto 
prazo para todos os membros da cadeia, constituiu a principal barreira para a adoção dos conceitos da gestão da cadeia de suprimentos. Numa tentativa de romper essa barreira, no final da década de 1990 surgiu o conceito do planejamento colaborativo (VICS, I998). O planejamento colaborativo tem como foco específico ações conjuntas entre as empresas participantes da cadeia de suprimentos, com o objetivo de reduzir os níveis de estoques e de melhorar os níveis de serviço (AROZO, 2000). Não obstante, a implementação de qualquer conceito ou filosofia de gestão administrativa envolve pessoas como usuárias de sistemas de informação, total ou parcialmente computadorizadas. Dessa forma, o fator crítico para o seu sucesso não é a barreira técnica, e sim a barreira psicológica. O fator humano é parte importante no processo (BARRAT, 2002). Esse tanto pode criar obstáculo, como agilizar a sua implementação e operacionalização.

Assim, partindo-se da premissa de que é possível estabelecer um relacionamento (ou associação) entre a colaboração dos funcionários e o desempenho do planejamento colaborativo, a pesquisa, objeto deste artigo, partiu do seguinte problema: qual é o grau de relacionamento que se evidencia entre a colaboração dos funcionários e o desempenho do planejamento colaborativo na Klabin S. A.? O objetivo é mapear a contribuição da colaboração dos funcionários no desempenho do planejamento colaborativo. Esse objetivo se justifica no argumento de que é importante para o gestor conhecer as origens da eficácia de qualquer conceito administrativo implementado na empresa, de maneira que evite o desperdício dos poucos recursos disponíveis em projetos cujos resultados ainda são duvidosos. Como exemplo disso, há as implementações das ferramentas eletrônicas de integração da cadeia de suprimentos, cujo investimento, em 2002, chegou a US\$ I9 bilhões. Contudo, poucas empresas atingiram os objetivos pretendidos (MENDES, 2003). O baixo desempenho das ferramentas eletrônicas de integração tem como causas a falta de confiança entre os parceiros de negócio, a necessidade de maior empenho do executivo principal e os conflitos internos à empresa (TEIXEIRA JUNIOR; PADUAN, 2002; MENDES, 2003).

\section{REFERENCIAL TEÓRICO}

A colaboração e o planejamento colaborativo são temas que pertencem às áreas de conhecimento de estudos organizacionais e da gestão de operações e logística. Assim, dado o caráter interdisciplinar desta pesquisa, a seguir, são apresentados os conceitos e teorias desenvolvidos nesses campos de conhecimento, com a intenção de obter o entendimento e o suporte necessários para a análise dos dados e resultados obtidos neste estudo. 
- ANÁLISE EXPLORATÓRIA DA COLABORAÇÃO DOS FUNCIONÁRIOS E DO DESEMPENHO... •

\subsection{COLABORAÇÃO}

As pressões do mercado para o fornecimento de melhores serviços e produtos com menores preços têm feito emergir, nos últimos anos, a consideração do elemento humano como um fator-chave para o planejamento colaborativo no contexto da gestão da cadeia de suprimentos. Esse fato pode ser observado nas páginas internas dos manuais de implementações de sistemas administrativos, corporativos como o SAP (2003) ou modulares como o CPFR (2000), em que as pessoas envolvidas são conclamadas a colaborar para o seu sucesso. Embora as empresas reconheçam ser a colaboração de seus funcionários um fator crítico para o sucesso da implementação, isso nem sempre ocorre com a facilidade desejada. Para os estudiosos desse campo de conhecimento, a colaboração é um conceito nebuloso, ligado à cooperação e contrastando com conflito, comportamento adverso e competição (KNIGHT, 2000). Na verdade, o processo de colaboração envolve um largo espectro de negociações, acordos e contratos (HERZOG, 200I). Humprey et al. (200I) observaram que relações colaborativas requerem confiança e comprometimento para uma cooperação de longo prazo, com desafios e divisão de riscos. Argumentam ainda que a formação efetiva do sucesso da colaboração necessita de uma comunicação eficiente em todos os níveis e de um compartilhamento de informação de modo contínuo inter e intraorganizacional. A entidade The Voluntary Inter-Industry Commerce Standards (VICS) (I998) menciona que os parceiros de negócios estão prontos para o planejamento colaborativo quando existe uma relação aberta de confiança por meio do compartilhamento de informações sensíveis, um forte comprometimento e um sólido trabalho em equipe.

Para o propósito deste estudo, colaboração foi entendida como uma deliberada cooperação nas relações de negócios, composta de três fatores-chave de "sucesso" no relacionamento colaborativo: confiança, comprometimento e trabalho em equipe (KNIGHT, 2000).

A confiança é um importante componente para a colaboração, porque somente pela confiança entre as pessoas é que se podem iniciar a interação e a comunicação, conduzindo-as na direção da colaboração (WILLIAMS, 200I; ZOLIN et al., 2002). Estudos acadêmicos revelaram que as economias geridas por sociedade em que há alta confiança desenvolvem-se muito mais rapidamente do que aquelas de sociedades de baixa confiança. No que se refere a aspectos individuais, quando se confia no outro, espera-se que este se comportará de maneira consistente com o nosso interesse (KUMAR; PADDISON, 2000).

O comprometimento é um estado psicológico em que há o envolvimento dos valores individuais e o desejo de permanecer envolvido com a organização, 
conforme argumenta Grant (2003). Isso já era uma preocupação de Fayol no início do século XX (SWAILES, 2002). Fayol acreditava que o interesse de uma organização deveria ter sempre a preferência em detrimento dos interesses individuais ou do grupo de empregados. Entretanto, não é uma tarefa fácil fazer que os indivíduos se tornem um agente com alto nível de comprometimento com a organização, especialmente numa sociedade como a ocidental, na qual o individualismo supera o coletivismo.

O trabalho em equipe é realizado por um grupo de pessoas caracterizadas por um elevado grau de sinergia. Essa sinergia combina e melhora os conhecimentos e as habilidades individuais dos membros do grupo para criar ou tomar decisões de melhor qualidade que as desenvolvidas individualmente (DAVIS et al., 200I). A ênfase no desempenho entre os membros do grupo é coletiva. Para Salas et al. (200I), existem muitos modelos conceituais, taxonomias e estudos empíricos demonstrando a importância do trabalho em equipe, embora ainda haja muita confusão a respeito do conjunto de conhecimentos e habilidades para a compreensão do trabalho em equipe.

Outra importante questão para esses três fatores-chave é a respeito de suas medidas de desempenho. A necessidade de medidas de desempenho repousa na idéia de que cada variável ou aspecto considerado só podem ser melhorados se eles forem mensurados, conforme defenderam Schroeder (I993) e Kaplan e Norton (1996). Várias dimensões para esses elementos-chave foram identificadas e medidas. As medidas variavam muito entre estudos efetuados. Por exemplo, para confiança, Kirsimarja et al. (2002) argumentavam que parecia ser uma tendência em que cada autor desenvolvia suas próprias medidas. Para Zolin et al. (2002), os fatores-chave confiança, comprometimento e trabalho em equipe tornam-se particularmente importantes quando tratam das atividades entre funções ou entre países. Por exemplo, a confiança torna-se difícil de ser desenvolvida quando a equipe de trabalho tem pouca oportunidade para interagir face a face, pois as pessoas devem confiar bastante na tecnologia para mediar suas interações, culturas e barreiras da linguagem. Entretanto, com o aumento da complexidade na administração das alianças que se estendem para as diferentes nações e culturas, a confiança e o comprometimento podem ser mais importantes nas alianças estratégicas internacionais do que quaisquer outros elementos (CULLEN et al., 2000).

Por fim, foi observado que o escopo deste estudo não foi aprofundar as questões teóricas concernentes à colaboração. O objetivo foi utilizar os conceitos já desenvolvidos para os relacionamentos interorganizacionais pelos autores que trabalham nesse campo de conhecimento e aplicá-los ao planejamento colaborativo no contexto da gestão da cadeia de suprimentos. 
- ANÁLISE EXPLORATÓRIA DA COLABORAÇÃO DOS FUNCIONÁRIOS E DO DESEMPENHO... •

\subsection{PLANEJAMENTO COLABORATIVO}

Em meio a tantos conceitos ou técnicas administrativas, os administradores de negócios precisam conhecê-los para aplicá-los de maneira eficaz. Produtividade, qualidade e reengenharia um dia tiveram o seu ápice. Quando a onda de cada modismo perde a sua força, um novo conceito toma o seu lugar. Para cada modelo de negócios da última moda, as organizações gastam milhões de dólares implementando e atualizando os seus sistemas de informação ou alterando suas estruturas organizacionais. Na trilha desses modismos, na década de iو80, as empresas asseguravam a sua vantagem competitiva somente se buscassem otimizar suas atividades internas. Na década seguinte, autores como Christopher (I992), Ballou (200I) e Bowersox e Closs (200I) argumentavam que, com as mudanças drásticas de natureza logística e a revolução da tecnologia da informação, as empresas deveriam repensar os relacionamentos empresariais com os seus parceiros.

A gestão da cadeia de suprimentos surgiu, então, como a grande solução, mostrando que os sistemas de informação corporativos como os Enterprise Resource Planning (ERP) eram de fato incompletos e não refletiam as novas necessidades demandadas pelo mercado daquele período. Embora o conceito original de gestão da cadeia de suprimentos tenha sido o de eliminar as barreiras organizacionais entre os parceiros comerciais com o objetivo de facilitar a sincronização da informação, mostrou também conter falhas por apresentar informações distorcidas. Isso conduzia às ineficiências operacionais, aos estoques excessivos, ao descontentamento dos consumidores, às perdas de receitas e, sobretudo, à falta de competitividade propagada para todos os membros da cadeia produtiva (DORNIER et al., I998).

Como a tendência dos últimos dez anos tem sido a aquisição externa de recursos, como a terceirização e a subcontratação, tornou-se prioritário para os integrantes da cadeia de suprimentos coordenar e integrar suas estratégias logísticas. Há o reconhecimento, cada vez maior, por parte de muitas empresas, de que a parceria e a cooperação proporcionam melhores resultados do que $o$ interesse próprio e o conflito (NALEBUFF; BRANDEBURGER, I996). Por isso, o planejamento colaborativo tem sido advogado pelos planejadores acadêmicos e profissionais como o novo paradigma na prática do planejamento (MARGERUM, 2002).

Em I998, focando especificamente a colaboração, a entidade The Voluntary Inter-Industry Commerce Standards (VICS, I998) publicou o Collaborative Planning, Forecasting and Replenishment (CPFR, 2000). O CPFR é um conjunto de regras e procedimentos para estabelecer padrões a fim de melhorar o fluxo de 
informações entre os parceiros de negócios. Foi originalmente concebido para envolver apenas um grande varejista e o seu fornecedor. Muitas empresas e provedores de serviços têm reconhecido que o CPFR também pode ser aplicado para os fabricantes e seus fornecedores.

Em conseqüência disso, uma organização pode ir além de um simples Enterprise Resource Planning (ERP) ao focar suas atividades internas e mover para as atividades externas, planejando colaborativamente com seus parceiros, reduzindo estoques da cadeia de suprimentos e disponibilizando os produtos de forma ágil na quantidade e local desejados. Dada a natureza dinâmica das cadeias de suprimentos, o desempenho do planejamento colaborativo é freqüentemente avaliado em termos de baixo custo de materiais, alta qualidade, redução do tempo de entrega, redução de estoques e acréscimo de vendas. Entretanto, isso somente é possível se as empresas colaborarem e partilharem informações e conhecimentos por meio de um conjunto de procedimentos estabelecidos de comum acordo (VICS, I998). É importante observar que a prática da colaboração é um fator relevante tanto dentro da empresa como além de suas fronteiras. De fato, muitos argumentariam que, antes de procurar colaborar com os parceiros externos, seria necessário assegurar o sucesso e a completa colaboração dentro da empresa (WHITE, I999).

\section{PROCEDiMENTOS METOdológicos}

\subsection{ESTUDO DE CASO}

Para verificar o relacionamento entre a colaboração dos funcionários e o desempenho do planejamento colaborativo, internamente à empresa conforme argumentou White (I999), procedeu-se à pesquisa de natureza exploratória, tendo como estratégia o estudo de caso. Essa escolha se deu porque representa uma maneira de investigar um tópico empírico, seguindo-se um conjunto de procedimentos pré-especificados (YIN, 200I). Para o estudo, selecionou-se por conveniência a empresa brasileira do setor fabricante de papel e celulose, a Klabin S.A., situada no Paraná. As razões para a escolha dessa empresa foram: a) tem uma representatividade significativa no setor produtivo brasileiro; b) aplica o planejamento colaborativo, por conseguinte, está envolvida em atividades como a reposição contínua, internação de materiais, contratos de parcerias e gestão de logística; c) trata-se de um estudo num setor em que o planejamento colaborativo não 
é o seu berço natural, pois a empresa escolhida não pertence ao setor varejista; e d) um dos participantes do estudo faz parte do corpo de funcionários, facilitando a aplicação, a revisão dos questionários e o acesso às informações secundárias, conforme a abordagem da observação participante (YIN, 200I).

Para responder ao problema de pesquisa, foi estabelecido como objetivo geral: mapear a contribuição da colaboração dos funcionários na melhoria do desempenho do planejamento colaborativo. Como objetivos específicos: a) medir transversalmente as variáveis da colaboração dos funcionários; b) medir longitudinalmente as variáveis do desempenho do planejamento colaborativo considerando a data atual e há um ano; e c) identificar outras evidências que corroborem os resultados obtidos nos itens a e b.

Assim, com base no referencial teórico e nos objetivos propostos, as variáveis foram assim consideradas:

- Independentes: quando há a colaboração dos funcionários. Nesse caso, as variáveis que compuseram a colaboração foram as candidatas a explicar as dependentes. Foram elas: a confiança, o comprometimento e o trabalho em equipe.

- Dependentes: quando há o desempenho do planejamento colaborativo. O planejamento colaborativo foi o fenômeno a que se propôs estudar. Esse fenômeno foi constituído por variáveis como a redução de estoques, a confiabilidade na entrega dos produtos e o congelamento da programação da produção.

Para atingir os objetivos propostos e, por conseguinte, responder ao problema de pesquisa, utilizou-se uma matriz de relacionamento (ou associação) que mostrava o grau de relacionamento entre essas duas variáveis: as independentes e as dependentes.

\subsection{CARACTERIZAÇÃO DA KLABIN S.A.}

A Klabin é uma companhia de capital aberto, constituída de ig plantas industriais: quatro de papel, onze de papelão ondulado e quatro de sacos e envelopes; além de áreas de reflorestamento nos Estados de São Paulo, Santa Catarina e Paraná. A principal unidade fabril, objeto deste estudo, foi fundada em I934 e está localizada no município de Telêmaco Borba, Paraná. A unidade compreende o maior complexo integrado florestal-industrial do País e incorpora tecnologias para o controle ambiental de sua atividade fabril no que concerne a emissões hídricas e atmosféricas e na geração de resíduos sólidos. Dentre os papéis fabricados, destacam-se o cartão dúplex para embalagem de líquidos 
e o Kraftliner, este utilizado na produção de caixas pela Unidade de Papelão Ondulado e também destinado à exportação. A Klabin atua no mundo todo, num mercado de alta competitividade, característico de produtos como papel e celulose. Auto-suficiente na produção de madeira e celulose, a Klabin opera com um dos mais baixos custos de produção em nível mundial e margens reduzidas de lucro, o que torna dispendioso o acúmulo de estoques para suportar as flutuações da demanda.

\subsection{COLETA DOS DADOS E SUJEITOS DA PESQUISA}

Para o estudo, coletaram-se dados primários e secundários. Os dados primários coletados na pesquisa foram obtidos por meio de entrevistas, utilizando-se de um questionário semi-estruturado composto de questões abertas e fechadas. Nas questões abertas, constituídas de oito perguntas, o entrevistado teve a liberdade de expressar suas percepções a respeito da colaboração e do desempenho do planejamento colaborativo, com o objetivo de identificar, qualitativamente, evidências de relacionamento. Foram desenvolvidas, ainda, questões fechadas com a finalidade de medir, quantitativamente, as variáveis da colaboração e do desempenho do planejamento colaborativo.

I) As variáveis quantitativas referentes à colaboração dos funcionários foram medidas transversalmente. A razão de adotar essa abordagem se deveu à impossibilidade de serem retroagidas. Entretanto, em uma pesquisa futura, as medidas das variáveis da colaboração poderão ser coletadas longitudinalmente, uma vez que já se tem o histórico do período anterior. Consciente dessa limitação, apresentaram-se aos respondentes quinze assertivas, sendo cinco delas para cada um dos três fatores-chave referentes a essa variável. A construção das assertivas baseou-se em Knight (2000) e Grant (2003), já indicados no referencial teórico. A utilização de cinco assertivas em cada fator-chave deveu-se, basicamente, às restrições de caráter operacional. Desejava-se um questionário não muito extenso e com um certo grau de robustez de conceituação teórica, de tal forma que se evitassem dúvidas quanto ao seu preenchimento. Para cada assertiva da colaboração, considerada como qualitativa ordinal, adotou-se uma escala do tipo Likert, com seis opções de respostas: I. Discordo fortemente, 2. Discordo moderadamente, 3. Discordo levemente, 4. Concordo levemente, 5 . Concordo moderadamente e 6. Concordo fortemente. Escolheu-se essa escala por sua capacidade de verificar o grau de concordância ou discordância com as assertivas apresentadas, mos- 
- ANÁLISE EXPLORATÓRIA DA COLABORAÇÃO DOS FUNCIONÁRIOS E DO DESEMPENHO... •

trando-se apropriada ao se medirem sutilezas de atributos de uma mesma categoria de fenômeno (SAATY, I99I).

2) As variáveis quantitativas referentes ao desempenho do planejamento colaborativo foram medidas longitudinalmente, em que se procurou comparar o desempenho de dois períodos, o atual e o de um ano atrás. A adoção dessa abordagem foi facilitada porque os dados sobre o desempenho do planejamento colaborativo são normalmente registrados nos relatórios gerenciais e operacionais, portanto possíveis de serem retroagidos. Foram formuladas dez variáveis, na forma de assertivas, com base no manual da entidade VICS (I998) e consideradas como as mais importantes para a Klabin. Essas variáveis (ou assertivas) foram consideradas quantitativas contínuas, graduadas em uma escala intervalar de nove pontos (de i a 9). A escolha de medidas quantitativas, contínuas e intervalares, se deveu ao seguinte fato: desejava-se que as variáveis assumissem valores fracionários em intervalos conhecidos. As variáveis, entre os intervalos, poderiam assumir, ainda, um conjunto infinito de valores possíveis. No caso deste estudo, os intervalos foram fixados entre I e 9, e, entre os intervalos, uma casa decimal após o número inteiro. Com a fixação desses intervalos, embora arbitrária, acreditou-se que, dada a natureza da pesquisa e o objeto estudado, poder-se-ia obter uma relação adequada entre a precisão (capacidade) e a acurácia (refinamento ou ajuste fino) da medida proposta em acordo ao argumento de Pereira (I999). Assim, foi solicitado aos respondentes que dessem uma nota ou grau de i a 9, com até uma casa decimal para cada assertiva apresentada. As notas deveriam ser atribuídas para a situação atual e para a de um ano atrás. Se o respondente tivesse menos de um ano de trabalho na Klabin, foi pedido que ele desconsiderasse a coluna correspondente aos dados de um ano atrás.

Para a coleta dos dados primários, foram escolhidos como sujeitos da pesquisa os gerentes, supervisores e coordenadores das áreas de compras, suprimentos, marketing, vendas e logística da Klabin. A escolha dessas funções se deu pelas seguintes razões: a) são cargos de gestão estratégica do planejamento colaborativo e b) sua posição na hierarquia organizacional com condição favorável para avaliar o desempenho do planejamento colaborativo.

Os dados secundários foram obtidos por meio da consulta dos documentos oficiais da Klabin, como propaganda institucional, jornais internos, relatórios gerenciais e relatório anual (balanço financeiro). 


\subsection{TRATAMENTO DOS DADOS}

Os dados primários obtidos por meio das questões abertas e os secundários coletados por meio dos documentos oficiais da Klabin foram tratados na forma qualitativa. Nas questões abertas, utilizou-se a análise de conteúdo (BARDIN, I977; YIN, 200I) que, por meio das descrições do conteúdo das mensagens escritas, procurou inferir conhecimentos inerentes à colaboração e ao planejamento colaborativo. Com relação aos documentos oficiais coletados, aplicou-se a técnica da análise documental (BARDIN, I977). Por meio dessa técnica, buscou-se encontrar, nos jornais internos, materiais de publicidade junto aos clientes e relatórios anuais, fatos convergentes na direção da colaboração e do planejamento colaborativo, contribuindo, assim, para a corroboração ou valorização das evidências pesquisadas.

Os dados primários coletados por meio das questões fechadas foram tratados na forma quantitativa. Embora, nesse estudo, a amostra (n) seja considerada de tamanho pequeno ( $5 \leq \mathrm{n} \leq 30$ respondentes), típico dos estudos de caso, procurou-se mostrar, por meio das técnicas estatísticas, os contrastes das medidas das variáveis coletadas. Entretanto, ressalta-se que, embora existam técnicas estatísticas para tratar de amostras de tamanho pequeno como a "t de Student" associada a dados paramétricos e o qui-quadrado $\left(\chi^{2}\right)$ tanto para dados paramétricos como para os não-paramétricos, para esse estudo foram utilizados:

- Para o desempenho do planejamento colaborativo, das estatísticas: média, como uma medida ou indicador do fenômeno estudado e da porcentagem de melhoria de desempenho do período atual em relação ao período de um ano atrás. Como os desempenhos foram coletados em uma base de dados quantitativos e contínuos utilizando-se uma escala do tipo intervalar, o cálculo das médias e a média da soma das médias dessas variáveis, identificada como intensidade média, não apresentaram dificuldades, pois os cálculos das médias e porcentagens puderam se utilizar da aritmética simples (COSTA NETO, 1977);

- Para a colaboração dos funcionários, das estatísticas: média, como uma medida (ou indicador) do fenômeno estudado e da confiabilidade ou consistência das medidas obtidas, segundo a abordagem de Pereira (1999) e Hair et al., (2005). Assim, para a média foram calculadas:

a) média das assertivas (variáveis) identificada como impacto das categorias segundo a sua progressão na escala ordinal. Como as assertivas (variáveis) foram constituídas da escala ordinal de categorias de Discordância/Concordância, pareceu sugestivo considerar que os intervalos entre as categorias eram regulares, de tal forma que a categoria "Discordo 
fortemente (DF)" pudesse corresponder ao valor numérico igual a I, "Discordo moderadamente (DM)" ao valor numérico igual a 2 e assim por diante. Com a codificação das categorias, que variaram de "Discordo fortemente (DF)" a "Concordo fortemente (CF)", estes, agora, poderiam assumir valores numéricos entre i e 6 . Dessa forma, chegou-se à composição de uma nova variável, em nível superior às variáveis (assertivas) originais. Entretanto, como as variáveis originais são medidas em seis categorias de intensidade, a nova variável não poderia ser uma simples contagem de presença ou ausência, sob pena de perda de informação (nesse caso, tanto um "Discordo fortemente" como um "Discordo levemente" dariam falsamente a mesma contribuição, significando ausência da Colaboração). Para contornar essa dificuldade, foram utilizados os códigos das categorias como pesos. O final é o surgimento de uma nova variável, denominada impacto, uma função linear das assertivas (variáveis) originais, representada pela fórmula:

$$
\text { Impacto }=\frac{[\text { freqüência }(\mathrm{DF}) \times \text { peso }(1)+\ldots+\text { freqüência }(\mathrm{CF}) \times \operatorname{peso}(6)]}{\text { frequiência total }}
$$

b) média da soma dos impactos, identificada como intensidade média. Como os impactos de diferentes tipos de variáveis (ou assertivas) estão sendo calculados, a fórmula do impacto das categorias pode ser simplificada para a soma desses impactos, segundo a fórmula:

Intensidade Média $=\frac{\sum \text { dos impactos de cada assertiva da categoria }}{\text { Número total de assertivas da categoria }}$

A nova variável (Intensidade Média) assim calculada é considerada um indicador aditivo. Um indicador é considerado de boa qualidade quando todas as suas medidas integrantes têm uma relação coerente entre si no esforço de medir o fenômeno considerado. Para medir se o indicador é composto de medidas originais com um nível de correlação bem estabelecido entre si, aceita-se o coeficiente Alfa $(\alpha)$ de Chronbach como a melhor representação do fenômeno. Assim, o Alfa $(\alpha)$ de Chronbach pode ser entendido como um coeficiente medidor da consistência interna entre as variáveis numa escala somada. Em geral, os limites mínimos de aceitação para o Alfa $(\alpha)$ de 
Chronbach são entre 0,6 e 0,7 (HAIR et al., 2005). Na abordagem de Yin (2001), o problema do tamanho da amostra considerado de tamanho pequeno nos estudos de caso pode ser sanado com o auxilio de outras evidências, como as análises qualitativas e observações diretas.

\section{ANÁLISE dOS dAdOS e RESULtAdos}

O estudo de caso compreendeu o período entre o último trimestre de 2003 e o primeiro trimestre de 2004. Foram entrevistados sete executivos das áreas de compras e suprimentos, logística, vendas e marketing. A análise dos dados e resultados foi dividida em duas partes: a primeira parte referiu-se aos dados primários coletados por meio das questões fechadas e a segunda parte, aos dados primários coletados por meio das questões abertas e secundárias, obtidas por consultas aos documentos oficiais da Klabin.

\subsection{ANÁLISE QUANTITATIVA DOS DADOS E RESULTADOS}

\subsubsection{COLABORAÇÃO DOS FUNCIONÁRIOS}

Na Tabela I, são mostrados os resultados da colaboração dos funcionários para o período atual.

Verifica-se, pela Tabela I, que:

a) A confiança e o comprometimento dos funcionários apresentaram a mesma intensidade média, o que foi seguido pelo trabalho em equipe com o valor igual a 4,3, 4,3 e 4,0, respectivamente, numa escala que variou entre i e 6 , cujo valor "I" correspondia ao pior resultado e o valor " 6 " ao melhor resultado.

b) O aspecto "comprometimento" com a assertiva "Comprometimento racional" correspondente à declaração "Para nós, aliança estratégica não é somente fazer parcerias, mas ter ações que, de alguma forma, proporcionem compensação econômica" foi o que apresentou melhor resultado, com a média (impacto) igual a 4,9. A segunda assertiva com o melhor resultado foi "Cético" do aspecto "confiança", correspondente à declaração "Minha empresa mantém-se informada e atualizada a respeito dos seus parceiros sem, no entanto, dividir todos os segredos ou informações estratégicas", com a média igual a 4,7 ; 
c) O aspecto "Trabalho em equipe", além de ser o pior elemento-chave da colaboração dos funcionários, evidenciou, ainda, a assertiva "Rígido controle", com o menor escore dado pelo impacto de valor igual a 3,6.

\section{TABELA I}

\section{IMPACTO (I), INTENSIDADE MEDIA E ALPHA $(\propto)$ DE CHRONBACH PADRONIZADO}

\begin{tabular}{|c|c|c|c|c|c|}
\hline \multicolumn{2}{|l|}{ CONFIANÇA } & \multicolumn{2}{|l|}{ COMPROMETIMENTO } & \multicolumn{2}{|l|}{ TRABALHO EM EQUIPE } \\
\hline Assertivas & (I) & Assertivas & (I) & Assertivas & (I) \\
\hline Cético & 4,7 & Autocontrole & 4,0 & Apoio mútuo & 3,7 \\
\hline Conflito & 4,6 & $\begin{array}{l}\text { Conhecimento dos } \\
\text { objetivos }\end{array}$ & 3,9 & Coordenação & 4,4 \\
\hline $\begin{array}{l}\text { Conhecimento dos } \\
\text { parceiros }\end{array}$ & 3,9 & $\begin{array}{l}\text { Comprometimento } \\
\text { emocional }\end{array}$ & 4,3 & $\begin{array}{l}\text { Estrutura para } \\
\text { cooperação }\end{array}$ & 4,0 \\
\hline Influência & 4,4 & $\begin{array}{l}\text { Comprometimento } \\
\text { racional }\end{array}$ & 4,9 & Foco no consumidor & 4,6 \\
\hline $\begin{array}{l}\text { Sentimento de } \\
\text { invulnerabilidade }\end{array}$ & 4,1 & Conexão & 4,6 & Rígido controle & 3,6 \\
\hline Intensidade média & 4,3 & Intensidade média & 4,3 & Intensidade média & 4,0 \\
\hline$\propto$ - padronizado & 0,54 & $\propto$-padronizado & 0,48 & $\propto$ - padronizado & 0,24 \\
\hline Geral $\quad \rightarrow$ & sidade & Média = 4,2 & & $\propto-$ Padronizado $=0$, & \\
\hline
\end{tabular}

Fonte: Dados dos autores.

Com relação à consistência interna das intensidades médias, verificou-se, pela Tabela I, que apenas o indicador geral dado pela intensidade média igual a 4,2 apresentou o coeficiente Alpha $(\propto)$ de Chronbach padronizado igual a 0,73 , isto é, acima dos limites aceitáveis, entre o,6 e o,7. Esse valor dá uma indicação de que a intensidade média obtida para o conjunto das assertivas tem uma boa correlação com as medidas originais. Para confiança, comprometimento e trabalho em equipe, a consistência interna do indicador dada pela intensidade média, os coeficientes de Alpha $(\propto)$ de Chronbach ficaram aquém dos limites aceitáveis. Uma das soluções para se conseguir um coeficiente dentro dos limites aceitáveis é aumentar o tama- 
nho da amostra ou buscar novas fontes de evidências. Nota-se, ainda, que foi utilizada a versão padronizada do coeficiente Alpha $(\propto)$ de Chronbach. Normalmente, o Alpha $(\propto)$ e Alpha $(\propto)$ padronizados têm valores muito semelhantes quando as medidas se utilizam de uma mesma escala e variâncias próximas (Pereira, I999).

Verificada a colaboração dos funcionários, cujas intensidades médias ficaram no lado concordante da escala, prosseguiu-se na verificação do desempenho do planejamento colaborativo.

\subsubsection{PLANEJAMENTO COLABORATIVO}

Na Tabela 2, são apresentadas as notas ou graus atribuídos ao desempenho do planejamento colaborativo, correspondentes ao período atual e ao período de um ano atrás.

\section{TABELA 2}

DESEMPENHO DO PLANEJAMENTO COLABORATIVO

\begin{tabular}{|c|c|c|c|c|}
\hline PERÍODO & UNIDADE & Atual & 1 ANO & $\%$ \\
\hline & & & ATRÁS & MELHORIA \\
\hline $\begin{array}{l}\text { Estoques de produtos } \\
\text { acabados }\end{array}$ & $N^{\circ}$ de dias & 7,3 & 7,2 & 1,4 \\
\hline $\begin{array}{l}\text { Estoques de matéria-prima e } \\
\text { componentes }\end{array}$ & $N^{\circ}$ de dias & 7,1 & 6,8 & 4,4 \\
\hline Entrega na data prometida & $\%$ ordens & 7,3 & 6,6 & 10,6 \\
\hline Entrega $100 \%$ dos itens & $\%$ ordens & 7,0 & 6,6 & 9,0 \\
\hline Aumento do faturamento & $\% \$$ & 7,7 & 7,4 & 4,1 \\
\hline Previsão de vendas & \% acurácia & 7,3 & 7,0 & 4,3 \\
\hline $\begin{array}{l}\text { Previsão de vendas de } \\
\text { componentes }\end{array}$ & \% acurácia & 7,0 & 7,2 & $-2,7$ \\
\hline Ordens sem programação & $\%$ ordens & 7,7 & 7,0 & 10,0 \\
\hline Planejamento da produção & $\begin{array}{l}\mathrm{N}^{0} \text { de dias de } \\
\text { congelamento }\end{array}$ & 7,8 & 7,0 & 11,4 \\
\hline
\end{tabular}


- ANÁLISE EXPLORATÓRIA DA COLABORAÇÃO DOS FUNCIONÁRIOS E DO DESEMPENHO... •

TABELA 2 (CONCLUSÃO)

DESEMPENHO DO PLANEJAMENTO COLABORATIVO

\begin{tabular}{lcccc}
\hline PERIODO & $\begin{array}{c}\text { UNIDADE } \\
\text { DE MEDIDA }\end{array}$ & Atual & $\begin{array}{c}\text { 1 ANO } \\
\text { ATRÁs }\end{array}$ & $\begin{array}{c}\% \\
\text { MELHORIA }\end{array}$ \\
\hline Planejamento da produção & $\begin{array}{c}\text { No de dias de } \\
\text { congelamento }\end{array}$ & 7,8 & 7,0 & 11,4 \\
\hline Acurácia da nota fiscal & $\%$ acurácia & $\mathbf{7 , 3}$ & $\mathbf{6 , 6}$ & 10,6 \\
\hline & Intensidade Média & $\mathbf{7 , 3}$ & $\mathbf{6 , 9}$ & $\mathbf{5 , 8}$ \\
\hline
\end{tabular}

*As unidades de medidas seguiram as recomendações de VICS (I998).

Fonte: Dados dos autores.

Observa-se, na Tabela 2, que as melhorias de desempenho mais relevantes foram: aumento do número de dias de congelamento da produção, entrega na data prometida, acurácia da emissão da nota fiscal e redução do número de emissões de ordens de produção sem programação com II, $4 \%$, I0,6\%, I0,6\% e I0,०\%, respectivamente. O pior desempenho do planejamento colaborativo foi a acurácia das previsões de vendas de componentes, em que, em termos porcentuais, houve uma regressão de $-2,7 \%$ em relação ao período de um ano atrás. Entretanto, utilizando-se do método de Aspin-Welch (COSTA NETO, I977) para amostra de pequeno tamanho, verificou-se que essa diferença em nível de $5 \%$ de significância é irrelevante. Assim, pode-se considerar que a acurácia da previsão de vendas de componentes permaneceu inalterada.

De forma geral, observou-se uma melhoria de $5,8 \%$ dos indicadores de desempenho do planejamento colaborativo entre a situação atual e a de um ano atrás.

\section{1.3 RELAÇÃO ENTRE A COLABORAÇÃO E O DESEMPENHO dO PLANEJAMENTO COLABORATIVO}

Efetuada a mensuração da colaboração dos funcionários e do desempenho do planejamento colaborativo, procurou-se verificar se havia algum tipo de relacionamento entre essas duas variáveis. Retirando-se os valores das intensidades médias referentes à colaboração e ao desempenho do planejamento colaborativo das Tabelas i e 2 e colocando-as em um gráfico de duas dimensões, com o eixo das abscissas (x) representando a colaboração e o eixo das ordenadas (y) representando o desempenho do planejamento colaborativo, obteve-se o Gráfico I, conforme é mostrado a seguir: 


\section{GRÁFICO I}

\section{COLABORAÇAOO VERSUS PLANEJAMENTO COLABORATIVO}

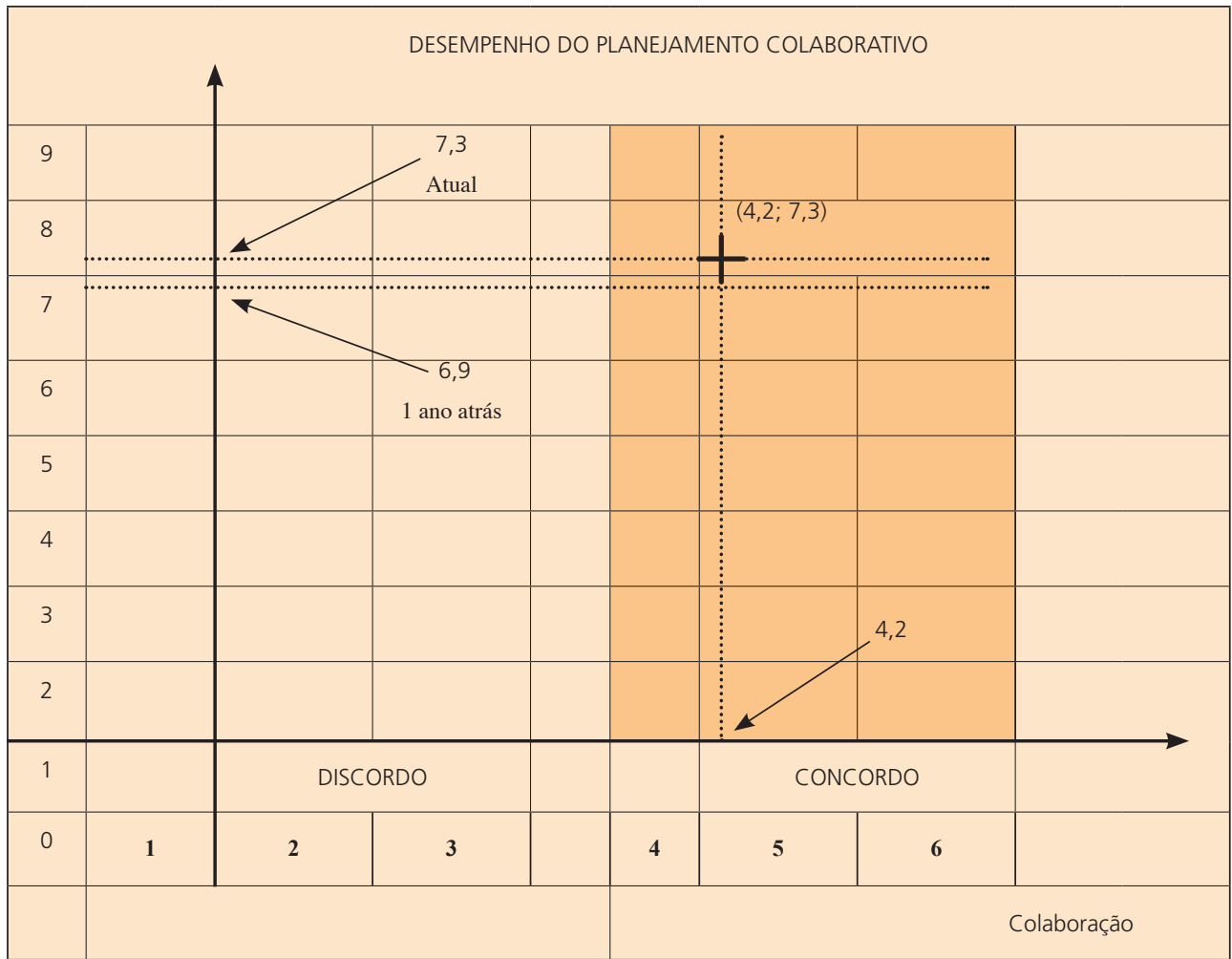

São observados no Gráfico I os seguintes pontos importantes: a) o eixo cartesiano está situado no ponto (I, I) e não no ponto $(\mathrm{O}, \mathrm{O})$, porque a escala de medida tanto para a colaboração como para o desempenho do planejamento colaborativo iniciou-se a partir da escala I (Discordo fortemente) e do grau ou nota I, respectivamente; b) a intensidade média do desempenho do planejamento colaborativo do período atual foi maior do que o do período de um ano atrás, com valores iguais a 7,3 e 6,9 , respectivamente, portanto uma melhoria $5,8 \%$ no desempenho em relação ao período anterior; c) a intensidade média da colaboração dos funcionários situou-se no lado "Concordante" da escala com um valor igual a 4,2; e d) na interseção das intensidades médias do período atual, correspondente ao ponto $(7,3 ; 4,2)$, tem-se a posição gráfica do relacionamento entre a colaboração e o desempenho do planejamento colaborativo da Klabin.

Parece óbvio, na visão dos autores citados no referencial teórico, estabelecer uma relação de causa e efeito entre essas duas variáveis, tendo como ferramenta 
estatística a regressão linear. Nessa abordagem, ao se aumentar a colaboração dos funcionários, o desempenho do planejamento também aumentaria. Não obstante, essa interpretação é prejudicada, visto que uma das variáveis não foi avaliada longitudinalmente.

Uma segunda abordagem é considerar a coleta dos dados referentes às variáveis dependentes e independentes, como um subgrupo de indivíduos ou objetos, e analisá-las, na data atual, transversalmente. Especificamente, por meio da técnica analítica da análise de agrupamentos (cluster analysis), a amostra de indivíduos (ou objetos) é classificada em um pequeno número de grupos mutuamente excludentes, com base nas similaridades entre as entidades. Para aplicar a análise de agrupamento, inicialmente se estabelecem as dimensões e os limites dos subgrupos da amostra. As dimensões, no presente estudo, são representadas pela colaboração dos funcionários e pelo desempenho do planejamento colaborativo. Os limites podem ser estabelecidos da seguinte forma: na colaboração, o escore podia variar entre i e 6 . Parece razoável estabelecer o valor igual a 3,5 [(I +6)/2] como um escore mínimo, a partir do qual qualquer valor acima desse escore representaria a evidência da colaboração dos funcionários. Aplicando a mesma idéia para o planejamento colaborativo cujo escore podia variar entre I e 9, sugere-se o valor igual a 4,5 [(I+9)/2] como um limite mínimo, a partir do qual qualquer valor acima desse limite representaria melhoria do desempenho do planejamento colaborativo. As dimensões e os limites podem ser apresentados na forma de uma matriz de dupla entrada, conforme é mostrado na Figura I, tendo na sua linha horizontal (abscissas) a colaboração dos funcionários e, na linha vertical (ordenadas), o desempenho do planejamento colaborativo.

Observa-se que o relacionamento (ou associação) entre a colaboração dos funcionários e o desempenho do planejamento colaborativo pode ser classificado em quatro subgrupos: I. baixa colaboração dos funcionários e alto desempenho do planejamento colaborativo, 2. alta colaboração e alto desempenho do planejamento colaborativo, 3. baixa colaboração e baixo desempenho do planejamento colaborativo e 4. alta colaboração e baixo desempenho do planejamento colaborativo.

Em resumo, utilizando-se a análise de agrupamento, a Klabin pode ser posicionada no Grupo "2", denotando uma empresa com alto grau de relacionamento entre a colaboração dos funcionários e o desempenho do planejamento colaborativo. Como extensão da análise de agrupamento, o resultado obtido pela Klabin pode ser utilizado para efetuar comparações com empresas similares.

Complementando a análise quantitativa, dado que a amostra do estudo foi considerada de tamanho pequeno, ensejou-se identificar outras fontes de evidência, conforme argumentou Yin (200I), para obter maior clareza ou mesmo corroborar ou refutar os resultados até aqui obtidos. 


\section{FIGURA I}

MATRIZ DE RELACIONAMENTO ENTRE A COLABORAÇÃO

DOS FUNCIONÁRIOS E O DESEMPENHO DO PLANEJAMENTO

COLABORATIVO

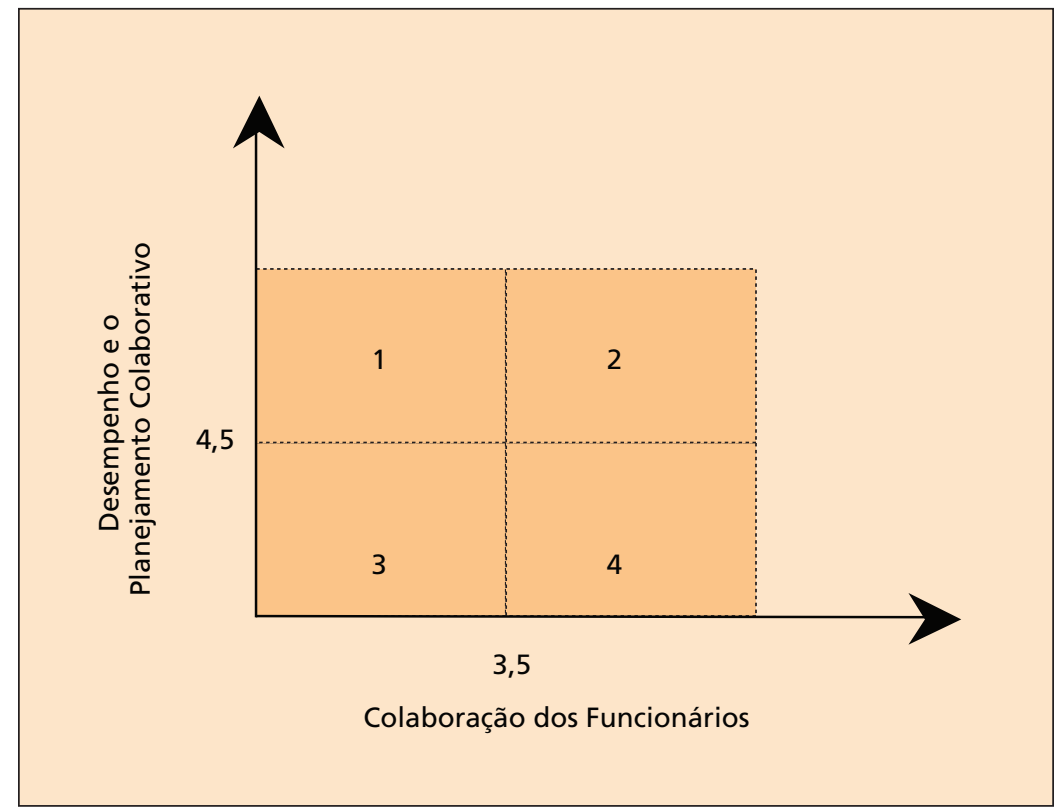

\subsection{ANÁLISE QUALITATIVA DOS DADOS E RESULTADOS}

Com base nos dados primários coletados por meio das questões abertas e secundárias obtidas em consulta dos documentos oficiais da Klabin para a análise qualitativa dos dados e resultados, obtiveram-se os seguintes resultados:

- Gerenciamento da cadeia de suprimentos: Para a Klabin, coordenar estrategicamente a cadeia de suprimentos, formada de diferentes negócios e gerenciada por diferentes pessoas, cada qual com seus próprios objetivos, é uma tarefa extremamente desafiadora. Nessa perspectiva, o consumidor final de papel e celulose, ao decidir fazer uma compra, dispara ações ao longo de toda a cadeia de suprimentos. O propósito da gestão da cadeia de suprimentos da Klabin é assegurar que os membros que compõem a cadeia produtiva tenham um comportamento cooperativo, intensa troca de informações, 
para que juntos obtenham sincronismo a fim de satisfazer o consumidor final. Esse sincronismo entre as empresas é de fundamental importância, pois evita a formação de estoques ao longo da cadeia, desonerando em grande parte os custos da produção. De uma forma simplificada, a cadeia de suprimentos da Klabin pode ser representada conforme o fluxo mostrado na Figura 2.

\section{FIgURA 2}

\section{FLUXO SIMPLIFICADO - PAPEL E CELULOSE}

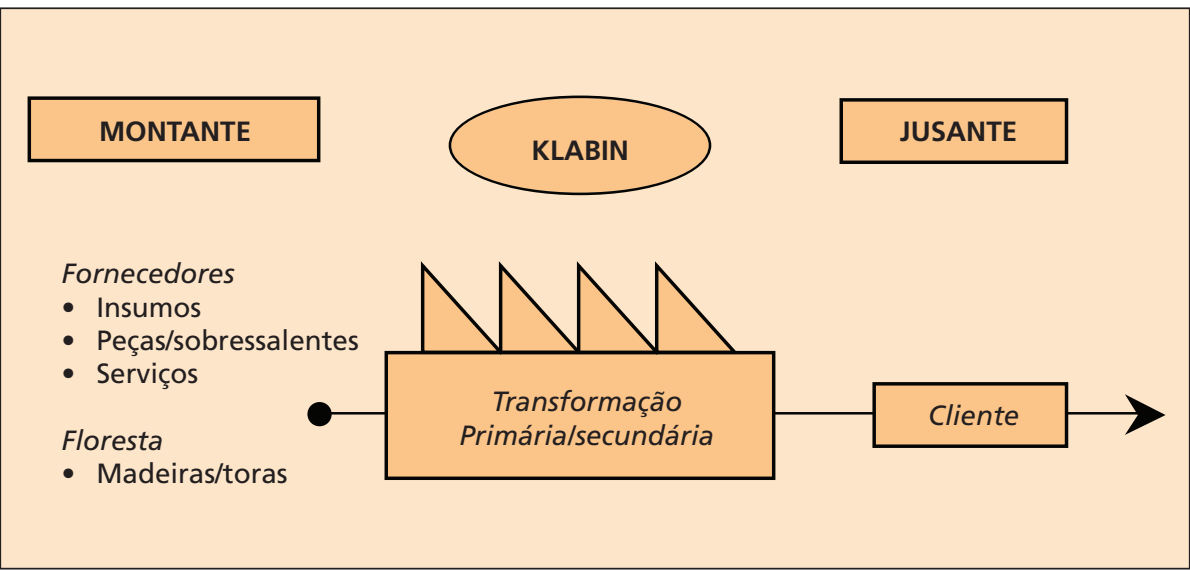

A jusante, a rede de distribuição da Klabin, em 2003, representou um faturamento de $\mathrm{R} \$ 2,7$ bilhões e exigiu esforços direcionados à melhoria da qualidade dos níveis de serviços, como redução dos prazos de entrega e qualidade dos produtos a preços baixos. Como exemplo disso, tem-se a prática do planejamento colaborativo com alguns grandes clientes, cujos pedidos se repetem. Assim, além das reposições contínuas dos estoques, o cliente é capaz de saber a sua posição a cada momento dos estoques, não necessitando do contato do vendedor para formalizar o pedido. Para tanto, a Klabin teve como estratégia o aumento da produtividade da frota de veículos na distribuição de produtos, a obtenção da consolidação das cargas e a redução de custos de transporte, uma vez que o dispêndio anual com fretes é significativo e o gerenciamento da logística tem um papel importante. 
A montante da cadeia de suprimentos, representando aproximadamente $40 \%$ do faturamento, a rede de suprimentos da Klabin, para os insumos e componentes essenciais para o produto final, teve como estratégia os relacionamentos de parceria de longo prazo com poucos fornecedores e médio grau de influência sobre eles, em razão da existência de uma baixa integração de seus processos produtivos. Trata-se de fornecedores de peças e sobressalentes para os equipamentos e os produtos químicos considerados insumos básicos para a fabricação de papel e celulose. Por sua vez, a rede de suprimentos para os produtos ou serviços secundários para os negócios da empresa busca a terceirização ou contratos de média duração. A adoção de uma estrutura organizacional de suprimentos do tipo híbrido, com um núcleo central responsável por contratos corporativos, normas, políticas de suprimentos e áreas de compras locais atendendo às necessidades específicas de cada unidade, favoreceu o gerenciamento colaborativo dos contratos e dos acordos de fornecimento de materiais de curto e longo prazos. A liberação de uma área interna à Klabin, para fornecedores de materiais secundários, como os de rolamentos, materiais elétricos, equipamentos de proteção individual (EPI) e ferramentas, teve como conseqüência a redução dos estoques e a melhoria das condições de negociação e, simultaneamente, dos níveis de serviços. Outro exemplo é a compra de soda cáustica, cujo tanque de depósito fica em área cedida pela Klabin, com reposição contínua do estoque.

Internamente à Klabin, cada operação tem como meta reduzir a sua própria complexidade e reduzir os custos de fazer negócios concretizados pela diminuição do ciclo operacional ou do tempo de atravessamento. Exemplos: uma operação cujo desempenho é marcado pela presença de defeitos freqüentes de qualidade no produto deve ser identificada, a fim de que se corrijam imediatamente as suas causas, para evitar a seqüência contínua de erros que podem chegar ao consumidor final. Deve-se evitar o desperdício do tempo de replanejamentos para compensar os erros cometidos. Devem-se padronizar e organizar as operações de transportes nas expedições com janelas de carregamentos predeterminadas, dando ao fornecedor (transportadoras) menor tempo de espera na unidade industrial, maior produtividade da frota e menor custo operacional. O que se procuram são os altos níveis de desempenho com tempo de atravessamento cada vez mais rápido, minimizando as flutuações da cadeia de suprimentos. O pressuposto básico disso tudo é obter uma maior visibilidade ao longo da cadeia para alcançar, simultaneamente, reduções de estoque e melhoria do nível de serviços.

- Recursos (ferramentas eletrônicas): Para efetivar a nova postura gerencial exigida pela gestão da cadeia de suprimentos, a Klabin iniciou, em 1999, implemen- 
tações de sistemas de gerenciamento de transporte, otimizador de rotas, Customer Relationship Management (CRM), Sistema Integrado de Comercialização e Produção (SICP) e de Enterprise Buyer Professional (EBP) para a atividade de compras pela internet, todos integrados ao sistema corporativo SAP. Esses sistemas tiveram como objetivos: a facilitação das práticas das compras eletrônicas, a cooperação entre os parceiros e a agilização dos fluxos de materiais ao longo da cadeia de suprimentos.

- Valorização dos funcionários: O maior desafio da gestão da cadeia de suprimentos, contudo, foi obter a colaboração dos funcionários desde a equipe de compras, vendas, programação, logística até a expedição. É evidente que a operação de sistemas de gestão em qualquer dimensão é um processo árduo, lento e mesmo doloroso. Departamentos inteiros são ceifados, outros são criados, tudo com o objetivo básico de manter-se competitivo. Dos funcionários, foram exigidos a disciplina e o cumprimento rigoroso dos procedimentos para obtenção do êxito dos desafios diários colocados à sua frente. Para tanto, a empresa proporcionou programas contínuos de treinamento em todas as áreas, com ênfase em microinformática e no autodesenvolvimento pessoal e profissional. Isso resultou em confiança e comprometimento dos funcionários que puderam vislumbrar um futuro dentro da organização, sem desconsiderar, no entanto, o fato de a unidade de negócio em estudo estar localizada numa região desprovida de poucas opções de emprego.

Em suma, com base nos dados primários referentes às questões abertas formuladas no questionário e nos dados secundários, coletadas qualitativamente, foi possível reforçar os resultados obtidos pelos dados coletados quantitativamente de que internamente à Klabin há evidências de um alto grau de relacionamento (ou associação) entre a colaboração dos funcionários e o desempenho do planejamento colaborativo.

\section{LIMITAÇÕES DO ESTUDO}

Neste trabalho não foram esquecidas as tradicionais críticas que o uso do método do estudo de caso provoca, tais como o tempo de execução e as inferências feitas dos resultados obtidos, e que apresentam deficiências de validade e confiabilidade para permitir qualquer inferência. A contra-argumentação diz que, embora a generalização não possa ser automática, ela pode ser feita por replicação em casos semelhantes, aumentando, dessa forma, a 
confiabilidade externa da pesquisa (YIN, 200I). De qualquer maneira, este estudo procurou atender aos padrões científicos para permitir a sua posterior replicação, de maneira que corrobore ou refute as evidências advindas dele (POPPER, I977).

\section{CONCLUSÕES E SUGESTÕES PARA PROSSEGUIMENTO}

Voltando-se ao objetivo principal deste estudo, verificou-se que a análise de agrupamento pode ser utilizada para examinar a associação entre a colaboração e o desempenho do planejamento colaborativo. Aplicando a análise de agrupamento à Klabin, verificou-se que o relacionamento entre a colaboração e o desempenho do planejamento colaborativo pode ser classificado como pertencente ao Grupo 2, isto é, trata-se de uma empresa de alta colaboração dos funcionários e alto desempenho do planejamento colaborativo.

Em relação aos objetivos específicos, verificou-se o seguinte:

- A evolução longitudinal do desempenho do planejamento colaborativo em que o escore da intensidade média, no período de um ano, passou de 6,9 para 7,3.

- Transversalmente, a colaboração dos funcionários se posicionou no lado concordante da escala com escores de 4,3; 4,3 e 4,0 para confiança, comprometimento e trabalho em equipe, respectivamente, evidenciando que o trabalho em equipe é o aspecto menos valorizado. Um estudo semelhante realizado em 2003, usando os mesmos dados desta pesquisa numa empresa inglesa do ramo químico, mostrou a confiança como o aspecto mais valorizado na colaboração. Discutir essa diferença é de fundamental importância, principalmente para as empresas multinacionais atuantes em países de diferentes culturas (ZOLIN et al., 2002; WILLIAMS et al., 2001).

- As evidências de práticas do planejamento colaborativo, como as atividades de e-commerce, implantação de softwares colaborativos, criação de janelas de carregamento de caminhões, contratos de reposição contínua de estoques e treinamento dos funcionários, corroboram os resultados obtidos quantitativamente.

Assim, pode-se concluir que é possível estabelecer graus de relacionamentos entre a colaboração dos funcionários e o desempenho do planejamento colaborativo, bem como compará-la aos seus concorrentes. Contudo, responder com certeza é uma tarefa extremamente desafiadora, porque dificilmente se saberá, com exatidão, o número de equações e o número de incógnitas envolvido no 
sistema colaborativo. Acrescente-se a isso o porte e a complexa estrutura organizacional da empresa estudada. Entretanto, uma evidência parece ter ficado clara: os desempenhos das variáveis que compõem o planejamento colaborativo não podem ser analisados pontualmente. Essa evidência tem que levar em consideração outras variáveis como as tecnológicas, de processo e de gestão, para evitar conclusões errôneas.

Para o prosseguimento de estudos, nessa linha, sugere-se: a) transformar esta pesquisa exploratória do tipo estudo de caso em descritivo, ampliando a amostra e estabelecendo as delimitações em termos de setores industriais; b) utilizar a matriz de relacionamento (ou de associação), conforme foi mostrada na Figura I, para visualizar a posição da empresa em relação aos seus concorrentes em termos de colaboração dos funcionários e desempenho do planejamento colaborativo; e, finalmente, c) introduzir outras variáveis como a atuação do executivo principal ou ambiente econômico favorável, para analisar o seu impacto no desempenho do planejamento colaborativo, aumentando, dessa forma, a confiabilidade interna da pesquisa (YIN, 200I).

Por fim, este artigo pretendeu oferecer uma contribuição para a prática dos administradores endereçada à valorização dos recursos humanos como um importante componente da vantagem competitiva empresarial. Em última análise, são as pessoas que realizam as mudanças. Projetos de sucesso são aqueles que envolvem soluções técnicas alinhadas com a aspiração de grande parte das pessoas envolvidas, reduzindo, assim, o desperdício de recursos que poderiam ser mais utilizados em outras atividades.

\section{REFERÊNCIAS}

AROZO, Rodrigo. CPFR - planejamento colaborativo: em busca da redução de custos e aumento do nível de serviços nas cadeias de suprimentos. Revista Tecnologística, São Paulo, ano VI, n. 6o, nov. 2000 .

BALLOU, Ronald H. Gerenciamento da cadeia de suprimentos. Planejamento, organização e logística empresarial. Porto Alegre: Bookman, 200I.

BARDIN, Laurence. L'analyse de contenu. Presses Universitaires de France, I977.

BARRAT, Mark. Don't forget the human element. England: Frontline Solution, June 2002. BOWERSOX, Donald J; CLOSS, David J. Logística empresarial. São Paulo: Atlas, 200 I.

CHRISTOPHER, Martin. Logística e gerenciamento da cadeia de suprimentos. São Paulo: Pioneira Administração de Negócios, I992.

COSTA NETO, Pedro Luiz de Oliveira. Estatística. São Paulo: Edgard Blucher, I977.

CPFR. Collaborative Planning Forecasting Replenishment. USA, 2000. Disponível em: <www. cpfr.com>. 
CULLEN, John B; JOHNSON, Jean L; SAKANO, Tomoaki. Success through commitment and trust: the soft side of strategic alliance management. Journal of World Business, USA, v. 35, n. 3, p. 225-226, 2000.

DAVIS, Mark M; AQUILANO, Nicholas J; CHASE, Richard B. Fundamentos da administração da produção. 3. ed. Porto Alegre: Bookman, 200I.

DORNIER, Philippe-Pierre et al. Global operations and logistics. New York: John Wiley \& Sons Inc., I998.

GRANT, Adam M. Having yourself committed: bridging between personal projects and organizational commitment. USA: Harvard University, 2003.

HAIR JUNIOR, Joseph F. et al. Análise de dados multivariados. Porto Alegre: Bookman, 2005.

HERZOG, Valerie Lynne. Canadá: Project Management Journal. 2000 International Student Paper Award Winner, March $200 \mathrm{I}$.

HUMPREY, P. K; SHIU, W. K; CHAN F. T. S. Collaborative buyer-supplier relationships in Hong Kong manufacturing firms. International Journal, v. 6, n. 4, $200 \mathrm{I}$.

KAPLAN, Robert S; NORTON, David P. Balance scorecard: translating strategy into action. USA: Harvard College, i996, p. 2I.

KIRSIMARJA, Blomqvist; SANNA Sundqvist; MARTTI, Soininen. Towards measuring inter-organizational trust. In: PROCEEDINGS OF EUROPEAN ACADEMY OF MANAGEMENT TRUST TRACK IN THE $2^{\text {ND }}$ ANNUAL CONFERENCE INNOVATIVE RESEARCH IN MANAGEMENT, May, Stockholm, Sweden, 2002.

KNIGHT, L. A. Learning to collaborate: a study of individual and organizational learning, and interorganizational relationships. UK: Journal of Strategic Marketing, v. 8, Issue 2, p. I2I-I38, June 2000. KUMAR, Ashok; PADDISON, Ronan. Trust and collaborative planning theory: the case of the Scottish Planning System. International Planning Studies, v. 5, n. 2, p. 210, 2000.

MARGERUM, Richard D. Evaluating collaborative planning. Journal of The American Planning Association, USA, v. 68, n. 2, Spring 2002.

MENDES, Maria Luisa. Gestão esperta, não estão entregando. Revista Exame, 20 ago. 2003.

NALEBUFF, Barry J.; BRANDENBURGER, Adam M. Co-opetition. Great Britain: Profile Books, I996. PEREIRA, Júlio César Rodrigues. Análise de dados qualitativos - estratégias metodológicas para as ciências da saúde, humanas e sociais. São Paulo: Edusp, Fapesp, I999.

POPPER, Karl Raimund. A lógica da pesquisa social. São Paulo: Cultrix, I977.

SAATY, Thomas L. Método de análise hierárquica. São Paulo: McGraw-Hill, I99I.

SALAS, Eduardo; BURKE, C. Shawn; CANNON-BOWERS, Janis A. Teamwork: emerging principles. International Journal of Management Reviews, v. 2, Issue 4, $200 \mathrm{I}$.

SAP. Systemanalyse and programmentwicklung. Sistemas, aplicações e produtos para processamento de dados. São Paulo, 2003. Disponível em: <www.sap.com/brazil>.

SCHROEDER, Roger G. Operations management, decision making in the operations function. 4. ed. USA: McGraw-Hill, I993. p. 757.

SWAILES, Stephen. Organizational commitment: a critique of the construct and measures. International Journal of Management Reviews, v. 4, Issue 2, June 2002.

TEIXEIRA JUNIOR, Sérgio; PADUAN, Roberta. A hora dos companheiros. Revista Exame, i3 nov. 2002.

VICS. The Voluntary Inter-Industry Commerce Standards. USA, I998. Disponível em: <www. vics.com>. 
WHITE, Andrew. The value equation: value chain management, collaboration and the internet. USA: Logility Inc., I999.

WILLIAMS, Michele. In whom we trust: group membership as an affective context for trust development. Academy of Management Review, v. 26, n. 3, 200 I.

YIN, Robert K. Estudo de caso: planejamento e métodos. Porto Alegre: Bookman, $200 \mathrm{I}$.

ZOLIN, Roxanne et al. Trust in cross-functional, global teams. Stanford University, USA: CIFE

- Center for Integrated Facility Engineering, 2002.

\section{TRAMITAÇÃO}

Recebido em 17/8/2005

Aprovado em 23/11/2006 


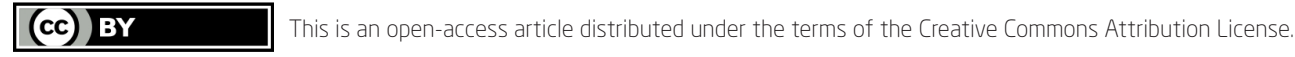

This paper may be copied, distributed, displayed, transmitted or adapted if provided, in a clear and explicit way, the name of the journal, the edition, the year and the pages on which the paper was originally published, but not suggesting that RAM endorses paper reuse. This licensing term should be made explicit in cases of reuse or distribution to third parties. It is not allowed the use for commercial purposes.

Este artigo pode ser copiado, distribuído, exibido, transmitido ou adaptado desde que citados, de forma clara e explícita, o nome da revista, a edição, o ano e as páginas nas quais o artigo foi publicado originalmente, mas sem sugerir que a RAM endosse a reutilização do artigo. Esse termo de licenciamento deve ser explicitado para os casos de reutilização ou distribuição para terceiros. Não é permitido o uso para fins comerciais. 